Recepción: 20 / 04 / 2017

Aceptación: 20 / 05 / 2017

Publicación: 15 / 06 / 2017

Ciencias de la Educación

Artículo de Investigación

\title{
Difusión de los recursos educativos abiertos (REA) para uso académico de profesores y estudiantes
}

\section{Dissemination of open educational resources (OER) for academic use of teachers and students}

Divulgação de recursos educacionais abertos (REA) para professores acadêmicos $e$ estudantes

\author{
Erick O. Guerrero-Zambrano ${ }^{\text {I }}$ \\ erick.guerreroz@ug.edu.ec \\ María M. Jaramillo-Campos II \\ maria.jaramilloca@ug.edu.ec \\ Sandra M. Zapata-Vega III \\ sandra.zapatav@ug.edu.ec
}

Correspondencia: erick.guerreroz@ug.edu.ec

I. Programador de Sistemas, Analista de Sistemas, Licenciado en Sistemas de Información, Magister en Comunicación Pública de la Ciencia y la Tecnología, Docente Universidad de Guayaquil, Guayaquil, Ecuador.

II. Ingeniero en Sistemas Computacionales, Magister en Administración de Empresas, Diploma Superior en Gerencia de Marketing, Docente Universidad de Guayaquil, Guayaquil, Ecuador.

III. Secretaria Ejecutiva en Sistemas de Información, Licenciada de Lengua Inglesa Especialización Lingüística y Literatura, Master in Teaching English As a Foreign Language, Docente Universidad de Guayaquil, Guayaquil, Ecuador. 


\section{Resumen}

Se difunde el uso de Recursos Educativos Abiertos (REA) en la Facultad de Ingeniería Industrial de la Universidad de Guayaquil, Ecuador que contienen una colección clasificada y ordenada de recursos educativos gratuitos en Internet, favoreciendo el acceso y fomentando el uso equitativo de estos elementos en América Latina y el mundo entero. Se realizó una investigación cuantitativa que indaga si los profesores y estudiantes universitarios tienen conocimiento de la existencia de estos recursos en Internet. El estudio determina que un alto porcentaje de profesores desconocen la existencia de ellos y no saben cómo alcanzarlos de forma directa, manteniendo la incertidumbre de que los elementos encontrados mediante buscadores tradicionales, no posean el aval y reconocimiento de instituciones académicas que garanticen la veracidad del contenido sin pagar por el acceso y uso de la misma, la difusión de los REA contribuye en la disminución del fenómeno conocido como "brecha digital" que existe en las poblaciones de los países con poco o nada acceso a la tecnología y a la información, donde las poblaciones universitarias diversas también se ven afectadas por el fenómeno.

Palabras clave: Recursos educativos abiertos; educación abierta; buscadores académicos; plataformas académicas; libre acceso; información libre. 


\begin{abstract}
The use of Open Educational Resources (REA) is dispersed in the Industrial Engineering Faculty of the University of Guayaquil, Ecuador, which contain a classified and ordered collection of free educational resources on the Internet, favoring the access and promoting the equitable use of these elements in Latin America and the whole world. A quantitative research was carried out to investigate whether the professors and the university students are aware of the existence of these resources in Internet. The study determines that a high percentage of professors are unaware of their existence and do not know how to achieve them directly, maintaining the uncertainty that the found elements through traditional search engines, do not have the endorsement and recognition of academic institutions that guarantee the veracity of the content without paying for the access and use of this resource,the difussion of OER contribute to the reduction of the phenomenon known as the "digital divide" that exists in the populations of countries with little or no access to the technology and information, where diverse university populations are also affected by the phenomenon.
\end{abstract}

Key words: Open Educational Resources, Open Education, Academic Searchers, Academic Platforms, Free Access, Free Information 


\section{Resumo}

o uso de Recursos Educacionais Abertos (REA), na Faculdade de Engenharia Industrial da Universidade de Guayaquil, Equador contendo uma substância classificada e ordenada recursos educacionais gratuitos na coleção do Internet espalha, facilitando o acesso e promover a utilização equitativa desses elementos em América Latina e no mundo. pesquisa quantitativa que investiga se professores universitários e alunos estão conscientes da existência desses recursos em Intenrnet foi realizada. $\mathrm{O}$ estudo determinou que uma elevada percentagem de professores não têm conhecimento deles e não sabem como alcançá-los directamente, mantendo a incerteza que os elementos encontrados através de motores de busca tradicionais não têm o aval eo reconhecimento de instituições acadêmicas que garantem a precisão do conteúdo sem pagar por acesso e uso dos mesmos, a difusão dos REA contribui na redução do fenômeno conhecido como "exclusão digital" que existe em populações de países com pouco ou nenhum acesso à tecnologia e informação, onde o várias populações universitários também são afectadas pelo fenómeno.

Palavras chave: Recursos Educacionais Abertos, Educação Aberta, Academic Search, Plataformas Acadêmicos, Open Access, Informação Livre 


\section{Introducción}

El uso de recursos de apoyo a la docencia ha tenido un crecimiento dinámico en los últimos años. Utilizar herramientas tecnológicas se ha convertido en prioridad entre los docentes y estudiantes; a manera de ejemplo, de las pizarras con tiza se trasladó al uso de pantallas digitales, de los textos impresos a libros digitalizados, de consultar en las bibliotecas a navegar en Internet para conseguir material electrónico y además las aulas han sido dotadas con infraestructura necesaria para poder soportar el uso de los recursos tecnológicos. De la misma forma, un nuevo movimiento se ha sumado a esta evolución donde la democratización del conocimiento es el punto de partida para el nacimiento de los recursos educativos abiertos (REA) como cuota innovadora. Estos elementos están llegando a formar parte de nuevos modelos pedagógicos para que sean promovidos, utilizados y compartidos entre estudiantes, profesores y con toda la comunidad educativa. La forma en que los REA son distribuidos es a través de portales o repositorios Web, que son iniciativas principalmente de universidades y a los cuales se han unido diversas entidades que cuentan con respaldo académico para su distribución de forma libre y abierta.

En la Web, se encuentra información de toda índole y podemos acceder a ella usando buscadores pero no podemos tener la certeza de que proviene de una fuente segura y confiable. Una forma verificar que la información a la que deseamos acceder es segura, es utilizar los repositorios pagados, donde encontramos artículos científicos, capítulos, cuadernos de memorias de congresos o libros especializados sobre la temática que se necesita pero muchas veces, la subcripción a ellos tiene un costo monetario medio-alto, cuyos valores son cubiertos por universidades, institutos de investigación o entidades de gobierno y cuyas licencias de uso expiran y deben ser renovadas periódicamente. 
Debido a la gran amplitud que posee la Web, hay muchos recursos eduactivos con respaldo académico y de licencia de libre acceso, que no se conoce porque simplemente no sabemos que existen y peor como llegar a ellos, presentándose como un problema de gran amplitud. El objetivo del artículo es mostrar los resultados de un estudio dirigido a docentes y estudiantes universitarios sobre la apropiación tecnológica y conocimiento de REA, la forma de llegar a ellos a través de la Web y difundir una lista de iniciativas REA que se muestran como otra alternativa académica para docentes y estudiantes, para que puedan ser utilizados libremente como recurso didáctico de estudio para generar nuevo conocimiento.

\section{Antecedentes}

El movimiento REA se inició en el 2001, en el Massachusetts Institute of Technology (MIT) donde fue creado el programa Open-CourseWare (OCW) que consistía en abrir al público en general el contenido de los cursos académicos que se impartían en las aulas de clase. Esto provocó que el interés por ellos vaya en aumento. Desde el tiempo que ha transcurrido de su creación, los contenidos abiertos para la educación se han encaminado por dos etapas de desarrollo: la inicial, centrada en ofrecer acceso a los contenidos y la más reciente interesada por el uso de agregarlas en las prácticas educativas.

Durante un taller sobre el uso el uso de cursos abiertos para países desarrollados, se utilizó por primera vez el término "recursos educativos abiertos" -en inglés, open educational resources (OER) - colocado en 2002 por la Unesco. Los OERs son los materiales y recursos educativos que pueden ser reutilizados para la docencia y el aprendizaje de forma gratuita (UNESCO, 2002). Los OERs son un denominador genérico que incluye cursos y programas curriculares, módulos didácticos, guías de estudiante, libros de texto, artículos de investigación, vídeos, podcasts, 
herramientas de evaluación, materiales interactivos (como simulaciones), bases de datos, software, aplicaciones (incluyendo aplicaciones móviles) y cualquier otro material educativo diseñado para uso en la enseñanza y el aprendizaje (OECD, 2007). En este sentido, no debe llevar a confusión con los elementos del proyecto OCW, que son materiales de alta calidad clasificados en cursos que conllevan una planificación, evaluación y que formarían parte de un tipo específico de OER. Se debe hacer una aclaración con respecto al aprendizaje online o e-learning, ni educación abierta, los REA no son iguales a estos elementos de aprendizaje mencionados. Los cursos e-learning se benefician de los REA, debido a esto no incluyen exclusivamente que correspondan a aprendizaje online. En efecto, muchos de los productos que se generan actualmente, aunque sean compartidos en formato digital, también se los puede encontrar en formato impreso (Unesco-IITE, 2011).

\section{Definiendo los Recursos Educativos Abiertos}

Basado en los argumentos anteriores se pueden definir los REA como recursos y materiales educativos con gratuidad y de libre acceso en Internet y la World Wide Web, compuestos de texto, video, audio, software, multimedia, entre otros, que poseen licencias libres para producir, distribuir y para usar en beneficio de la educación a nivel mundial. También se utilizan, licencias abiertas que se fundamentan en el marco existente de los derechos de propiedad intelectual, tal como vienen definidos en los correspondientes acuerdos internacionales, y respetan la autoría de la obra.

Las características de REA son:

El acceso. El recurso está disponible para su fácil localización y que se pueda utilizarn en cualquier momento.

Es sostenible. Los cambios de versiones no afectan la usabilidad. 
Es interoperable. El recurso se acopla y se interconecta con diferentes herramientas de hardware

La reutilización. El recurso puede modificarse y utilizarse en diferentes aristas de aprendizaje

Los metadatos. El recurso está indexado, almacenado, se busca fácilmente y se accede a el.

El uso de los REA contribuye a contrarestar a lo que los expertos denominan "brecha digital", en el cual están sumergidos las personas que no poseen acceso a la tecnología ni a la información, debido a que la difícil localización geográfica de ciertos sectores hacen que no sea posible llegar a ellos; comunidades en la pobreza es el común denominador u otro factor como de importancia como el "analfabetismo digital" debido a la falta de educación en tecnología de los pobladores. Pero este analfabetismo no solamente comprende que no se tenga acceso a las herramientas o no conocer cómo funcionan, sino también al desconocimiento de nuevos productos o recursos que son generados por entidades educativas bajo criterios que permitan tener credibilidad de alta calidad, confiabilidad y legalidad, y que tanto, estudiantes, profesores y comunidad académica desconoce de su existencia.

\section{Importancia}

Los REA se vuelven importantes en las entidades educativas, aunque su principal acogida ha sido las instituciones de primaria y secundaria, también su reputación y la garantía de contenidos hacen que sean utilizados cada vez más en universidades (Edrene, 2009). En países como México se han implementado repositorios para el uso público como Temoa (Mortera, 2010). Los repositorios europeos almacenan el 30.8\% de recursos educativos abiertos (Royster, 2011) en donde las universidades de España se han sumado significativamente desarrollando y colaborando con la 
Difusión de los Recursos Educativos Abiertos (REA) para uso académico de profesores y estudiantes

construcción de repositorios, llegando al 50\% de instituciones de educación superior en un total de 29 que apoyan el uso de REA (Abadal et al, 2009), entre las cuales se destaca la universidad de Alicante, obteniendo un premio a la excelencia otrogado por el OCW Consurtium, y la iniciativa Oberta en Abierto de la Universidad de Oberta de Cataluña (UOC) que posee reconocimiento y puntuación muy alta en el ranking de directorios abiertos Melibea. Varios estudios coinciden en que los REA aportan beneficios significativos para docentes, estudiantes, instituciones y público en general (GESER, 2007; Ferrán et al, 2007; Unesco, 2011).

El comportamiento positivo de docentes apoyando los repositorios, inclusive en la preservación, reutilización y creación de nuevos recursos, ha sido notable (Gadd, 2008). Los motivos en los que se enfatiza la planta docente es mejorar la docencia, trabajrr más fuerte en la motivación estudiantil y con ello, crear vínculos directos para con la insitución (Bates, 2007).

También existe una resistencia para no contribuir con REA: la resistencia al cambio, la falta de incentivos y la inexistencia que los repositorios existen realmente (Bates, 2007) a lo que se suma, el no saber usar la tecnología, al salir de la zona de confort usando lo mismo por mucho tiempo y la escasa destreza en el uso de las herramientas (Caris, 2004) y no poder ser capaz de encontrar los recursos en la Web ni tampoco poder reutilizarlos (Harley, 2008). Los estudios actuales están centrados en analizar el uso de REA, evaluando la utilidad y la práctica que le dan los usuarios y haciendo que los docentes integren los contenidos en las aulas (OPAL, 2011) debido a que aun no se ha llegado, a que los REA, sean utilizados constantemente.

En lo que se refiere al ámbitos financiero, debido a los ajustes y recortes de dinero, muchas veces impide poder contratar o mantener las licencias de uso de las plataformas académicas 
privadas en las entidades educativas, los REA se han convertido en una buena opción, para poder mantener y soportar dichos recortes presupuestarios (Olcos, 2007) .

Otro de los objetivos que se desea conseguir con el uso de REA es que se conviertan en palabras de uso diario, en conversaciones habituales entre profesores y alumnos, facilitando la difusión del aprendizaje y la mejora constante de la calidad tanto en la docencia como en el aprendizaje y la didáctica y no solamente de forma indivual como institución, al contrario, que pueda permitir el acceso a lo largo de toda la vida en todas partes del mundo, contribuyendo a la integración de la educación en todos los niveles.

Finalmente, los REA poseen un potencial que aún no ha sido explotado por completo, aún se encuentra en ese proceso, de querer convertir la educación, en algo al que todos puedan tener acceso y con ello, poder hacer un giro de 180 grados a la enseñanza y el aprendizaje. Esa mina llena de conocimiento que aún no se extrae, es hacer que dichos recursos puedan compartirse con total facilidad a través de Internet. Si esto se logra, transformaría totalmente la forma de generar conocimiento en la formación académica de los estudiante y en el refuerzo investigativo de los profesores, en la que Mary-Lou Fordware, afirma que el conocimiento crece cuando se lo comparte con los demás, convirtiendo a las personas, no solamente en consumidores de recursos, sino como verdaderos productores y adaptadores de conocimiento, cambiando así la cultura educativa de la sociedad.

\section{Planteamiento del problema}

Basado en lo descrito anteriormente se realizaron a preguntas de sondeo para obtener datos iniciales y en la revisión de evidencias físicas encontradas como las descripciones de elementos de clases tales como silabo, bibliografía principal y complementaria, se logró evidenciar que los 
profesores y estudiantes no conocen los repositorios donde se almacenan los recursos, ni como acceder a ellos.

Por lo tanto, es indispensable conocer el nivel de conocimiento y de acceso a los Recursos Educativos Abiertos (REA) por parte de los docentes y estudiantes.

El objetivo planteado es determinar el grado de conocimiento y accesibilidad de dichos recursos, y determinar las preferencias de profesores y estudiantes sobre las fuentes de información elegidas en la Web para realizar investigaciones académicas.

\section{Materiales y métodos}

El alcance que tiene este estudio es de identificar el grado de información y consumo sobre Recursos Educativos Abiertos (REA) de docentes y estudiantes de la ciudad de Guayaquil, en el año 2016. Teniendo en cuenta las posibles causas por las que conocen o desconocen esta herramienta digital, esta investigación proporcionará información relevante para las diferentes estrategias comunicacionales que se podrían proponer para la divulgación oficial y efectiva de REA.

La investigación a realizada fue dirigida para los estudiantes y docentes de las siguientes universidades:

Escuela Superior Politécnica del Litoral

Universidad Laica Vicente Rocafuerte

Universidad Estatal de Guayaquil

Con estos datos se podrá implementar mecanismos efectivos de comunicación, como también dejar en evidencia la importancia tanto para estudiantes y profesores, el uso necesario de los REA en las universidades del Ecuador. 


\section{Definición de la población}

Se define como población o universo al conjunto de elementos que van a ser analizados en la realización de un experimento. Dependiendo del número de observaciones que compone una población, esta puede ser finita o infinita. Aun en el caso de una población finita, los conjuntos de elementos podrían llegar a ser lo suficiente mente grandes como para que en la experimentación no puedan ser considerados todos ellos. En el caso de esta investigación la población objetivo será los estudiantes y maestros universitarios de la ciudad de Guayaquil.

\section{Definición de la muestra}

En el caso de que no sea posible la observación de toda la población debido a que es una población infinita que es imposible cubrir el análisis de todas las observaciones que la componen o es una población finita pero suficientemente grande que en la práctica, el coste y el tiempo que demandarían serian altos, entonces en situaciones como estas se debe trabajar con subconjuntos de observaciones de la población. Estos subconjuntos se los denomina muestra.

\section{Tipos de muestreos}

Entre los tipos de muestreos que se pueden utilizar como técnica de muestreo se encuentra, el tipo de muestreo probabilísticos, no probabilísticos y cuasi probabilístico. La diferencia fundamental entre estos procedimientos utilizados para seleccionar la muestra, es que se relaciona la probabilidad para seleccionar a los elementos que conformaran la muestra. Los muestreos probabilísticos son planes de selección de observaciones que se basan en el principio de selección aleatoria. En la práctica esto se refiere a que todas las observaciones de la población tienen una probabilidad conocida y distinta de 0 de pertenecer a la muestra. La aleatoriedad no es atributa de una muestra, sino del proceso de selección utilizado. 
Existen varias técnicas de muestreo probabilístico. Las diferencias entre ellas tienen que ver con la manera en que se escogen las observaciones u con la precisión de las estimaciones. Hay que recalcar que un diseño de muestra se puede tener más de una técnica de selección de observaciones. Se explican a continuación las técnicas de muestreo probabilístico utilizadas en esta investigación:

Muestreo Aleatorio Simple (MAS).- Esta técnica de muestreo se caracteriza porque la selección de la muestra se la hace de un listado de la población haciendo equiprobable la selección de una observación de esta última. Esta técnica es un procedimiento de muestreo básico y fundamental de otras técnicas.

Muestreo Estratificado.- Esta técnica consiste en la división de la población objetiva previamente en clases o grupos que se asumen homogéneos respecto a las característica a estudiar. A cada uno de los estratos seleccionados se le asigna una cuota que determinara el número de miembros del mismo que contendrá la muestra. Dentro de cada estrato el muestreo se lo hará mediante muestreo aleatorio simple.

Dentro del muestreo estratificado, existen dos técnicas de muestreo el cual se eligen en función de la cantidad de elementos:

Asignación proporcional. - El tamaño de la muestra en cada estrato es proporcional al tamaño de la población.

Asignación óptima. - El tamaño de la muestra va a depender de la variabilidad de los datos de cada estrato, es decir se incrementará la muestra de un estrato si en el mismo presenta más variabilidad. Para esto es necesario un conocimiento previo de la población.

Para fines de esta investigación se estudiaría por separado las opiniones de los estudiantes y los docentes, en la cual se asume que entre estos estratos se puede haber cierta homogeneidad. Por otro lado, dentro de esta técnica de muestreo estadística se considerará la asignación proporcional, 
debido a que no se tiene conocimiento de antemano de la población a estudiar. Para cada estrato se utilizó la fórmula de cálculo de tamaño de la muestra para población infinita debido a la escaza información de la población estudiantil como de los docentes de las universidades seleccionadas.

Para estimar el tamaño de la muestra necesaria para el cálculo de cada estrato se debe de aplicar la siguiente fórmula:

$$
n=\frac{z^{2} p q}{B^{2}}
$$

Donde $n=$ Tamaño de la muestra,

$z=1,645$ para el $90 \%$ de confianza 1,96 para el $95 \%$ y 2,56 para el $99 \%$

$p=$ Frecuencia esperada del factor a estudiar

$q=1-p$

$B=$ Precisión o error admitido

Según el cálculo del tamaño de la muestra a un nivel de confianza del 90\%, una probabilidad del $50 \%$ de éxito que la población objetivo (docentes y estudiantes) utiliza Recursos Educativos Abiertos $(\mathrm{p}=50 \%)$ y para una probabilidad de fracaso o de que no los utiliza $(\mathrm{q}=50 \%)$, el tamaño de la muestra $n=270,60$. De esta muestra calculada, la asignación para los estudiantes es del $80 \%$ que representa 220 encuestas estudiantes y para los docentes se asigna el $20 \%$ que representa 50 encuestas a docentes la desgloso para el estudio de campo de cada universidad que se detalló anteriormente.

En lo que respecta a la encuesta, se realizó el diseño de dos encuestas en la cual, una fue dirigida para estudiantes y la otra para docentes, ya que los fines de estos son distintos, las fuentes y sus hábitos de consumo de información no son necesariamente los mismos. 
Difusión de los Recursos Educativos Abiertos (REA) para uso académico de profesores y estudiantes

\section{Resultados}

\section{Docentes}

Desde el punto de vista de los docentes encuestados, todos hacen investigaciones en la web el cual el 67,1\% utiliza el buscador de Google para realizarlas. El 90\% de los docentes envía deberes a sus estudiantes por medio de la Internet, el cual el 91,8\% de estos mismos, entrega por lo general bibliografías o enlaces de páginas web con respaldo académicos a sus alumnos. Como ya se mención anteriormente, estos profesores realizan investigaciones utilizando la Internet, de los cuales el $62,5 \%$ conocen sobre motores de búsqueda académicos, como también el $70 \%$ de estos tienen noción sobre las TICS. Por otro lado, considerando lo explicado anteriormente, solamente el $50 \%$ de los profesores conocen sobre los Recursos Académicos Abiertos en la Web.

Con respecto al sustento académico de la información "educativa científica", solamente el 42\%, de los cuales el $75 \%$ de estos creen que es de confianza absoluta o tienen sustento académico. Sin embargo, un $15 \%$ cree que existe la posibilidad de que sea información manipulada. Por otro lado el $58 \%$ restante de profesores que mencionaron que no hay sustento académico de la información "educativa-científica", mencionaron razones de que puede ser información fraudulenta. Entre estas dos razones representa la opinión del 96,4\% de estos profesores.

Los docentes también contestaron que están de acuerdo en conocer nuevos recursos de educación en un 98\%. A demás los docentes creen que los enunciados principales de las TICs son el acceso a bibliotecas digitales, acceso a Internet y experiencia con estudiantes en un 37,8\%, 29,4\% y 27,7\% respectivamente. En cambio, para los Recursos Educativos Abiertos, los docentes creen que se refiere a software educativo en un $26,1 \%$, comunidades educativas en línea en un $23,9 \%$ y que tienen artículos científicos en un 23,2\%. El 95,9\% de los docentes cree que estos nuevos recursos de información si fomentarían el pensamiento crítico y la búsqueda de soluciones creativas ante los 
problemas. Los docentes considero de manera imparcial algunas páginas web como recursos Educativos Abiertos, como Wikipedia, Monografías, Slide Share, entre otros.

\section{Estudiantes}

Los estudiantes que fueron encuestados, provienen de la Universidad de Guayaquil que representa el 36,82\% (81 estudiantes), de la Escuela Superior Politécnica del Litoral con el 35,91\% (79 estudiantes) y de la Universidad Laica Vicente Rocafuerte (60 estudiantes). La edad promedio de estos es de 21 años aproximadamente. El 39,06\% siguen las carreras de Economía, Diseño gráfico, Medicina y Arquitectura, el resto de estudiantes siguen otras carreras, pero su representación es mínima. El 34,4\% son de primer año, 32,1\% de segundo año y el 27,5\% de tercer año. La mayoría es del sector norte es decir el 46,6\% de los estudiantes encuestados, seguido con el $26,5 \%$ que provienen del sur.

Las fuentes que utilizan los estudiantes para realizar investigaciones, generalmente provienen de Internet con el 79,9\%, seguido por bibliotecas con el 17,8\%. El 83,10\% de los estudiantes acuden a estas fuentes de información. El 78,08\% de los estudiantes utilizan el Internet diariamente. El $46,58 \%$ le destina de 2 a 4 horas y el 26,03\% le dedica entre 1 a 2 horas. En promedio los estudiantes dedican el 52\% de su tiempo para sus investigaciones académicas y el 93,09\% de ellos, utiliza Google como el buscador de preferencia, de los cuales el 89,58\% lo califico de muy bueno o excelente.

Las páginas web que utilizan con mayor frecuencia los estudiantes son Wikipedia con el 38,8\% y otras que representas páginas web como AltaVista.com, Rincón del Vago.com, Taringa.com, Monografias.com. El 93,5\% de los estudiantes consideras que existe una variedad de información académica, el cual también están dispuesto a conocerlas en un 91,2\%. El 61\% de los estudiantes no sabe acerca de los recursos académicos en la Web, el cual se constata con apenas un 
$14,7 \%$ de estos mismo que han ingresado al Google académico, $11 \%$ que ha ingresado al Educational Youtube. Sin embargo, el 57,14\% de los estudiantes al menos tienen conocimiento sobre bases de datos de revistas o artículos científicos.

\section{Discusión}

Al revisar los resultados obtenidos y relacionarlos con los objetivos planteados, se evidencia que el $67,1 \%$ de profesores hace investigación por Internet y no solamente, usando libros físicos con $32.9 \%$, en cambio los estudiantes lo usan la Web un 79,9\% y los que usan la biblioteca física es 17,8\%. Con respecto al conocimiento de REA, 50\% de los profesores encuestados no tiene conocimiento de ellos con respecto al $61 \%$ de estudiantes y en lo que respecta a las preferencias de búsqueda, los estudiantes utilizan en su mayor parte páginas Web que no poseen respaldo académico y cuya información es dudosa en un $38.8 \%$ y los que no conocen buscadores académicos $46.3 \%$ con respecto a los profesores que si los conocen con un $62.5 \%$.

Los docentes y estudiantes, en su gran mayoría, afirma en estar de acuerdo en conocer nuevas tecnologías que les ayuden en sus labores diarias, debido a que no conocen la existencia de REA pero usan el Internet para sus tareas e investigaciones y no conocen la forma de llegar a ellos y que estos recursos Web posean el respaldo académico necesario para poder utilizarse de forma confiable y segura.

\section{Conclusiones}

Un alto porcentaje de docentes y estudiantes usa el Internet como herramienta de investigación, pero un bajo porcentaje de ambos no tiene conocimiento de la existencia de REA. Las razones por las cuales los desconocen son debido a que no saben qué son, para que sirven, tendrán el respaldo académico que ellos requieren, les servirá para sus tareas diarias o para sus investigaciones. 
Uno de los objetivos de este trabajo es difundir a la sociedad en general, pero en especial a la comunidad académica, una lista de los sitios Web académicos, a los que se pueden acceder, donde la información está respaldada por universidades o centros de estudios formales, revistas científicas e institutos de investigación y que posee licencia de acceso libre para su difusión.

A continuación, se muestra una lista de portales Web de Recursos Académicos Abiertos o de Libre Acceso para conocimiento de docentes, estudiantes y público en general, donde encontrarán materiales de todas las áreas de la ciencia, de diversas temáticas y en varios idiomas:

https://ocw.mit.edu/index.htm

http://www.temoa.info/

https://eric.ed.gov/

http://www.namcol.edu.na/

http://www.open.edu/openlearn/

http://open.uct.ac.za/

https://www.oercommons.org/

http://www.iskme.org/

http://www.mdx.cat/

http://agrega.educacion.es/

http://www.teachingandlearning.ie/ndlr/

https://www.learntechlib.org/

http://www.oerafrica.org/

http://ocw.aca.ntu.edu.tw/ntu-ocw/

http://www.fetp.edu.vn/en/

http://www.oerafrica.org/ http://mit.ocw.universia.net/

http://www.tesisenred.net/

http://www.csuc.cat/es

http://www.euscreen.eu/

http://www.edutubeplus.info/

https://www.youtube.com/education

http://archive.tlt.psu.edu/mto/

http://www.edu365.cat/

http://www.ccma.cat/tv3/merli/

https://www.jisc.ac.uk/

https://www.merlot.org/merlot/index.htm

http://www.edna.edu.au/

https://www.openeducationeuropa.eu/en

https://ageconsearch.tind.io/

http://www.berkleeshares.com/

http://discovered.ed.ac.uk/ 
http://www.scielo.org/

Los materiales de los portales web del listado que se muestra, son de acceso libre, es decir, que se usa la herramienta de búsqueda del portal, se identifica el tema y se accede al recurso mediante un clic para descargarlo. Tampoco es necesario registrarse como cliente en el portal Web o acceder mediante una red social.

\section{Trabajo futuro}

Una vez reconocido la importancia de los Recursos Educativos Abiertos y de conocer portales Web donde acceder a los materiales, pasando por un proceso de selección de ellos, clasificándolos por asignaturas y por líneas de investigación definidas en cada una de las carreras de las universidades, podrán ser incluidos como material complementario de estudio en el sílabo de las asignaturas o tomados como referencia para proyectos integradores de cátedras o para continuar estudios y proponer temas nuevos de trabajos de titulación porque la calidad de la información que contienen es buena como los recursos que poseen licencia de pago. Una vez implementados en el sílabo, se puede medir de forma cualitativa o cuantitativa, el grado de aceptación del recurso para tener una aproximación de su utilidad y comprensión, haciendo evaluaciones de su contenido en tareas, pruebas parciales, exámenes o proyectos de curso.

\section{Agradecimiento}

El estudio se realizó gracias a la colaboración de los profesores Lcdo. Erick Guerrero, MSc., Ing. María Mercedes Jaramillo, MAE. y la Lcda. Sandra Zapata, MSc. y por el respaldo de la Facultad de Ingeniería Industrial de la Universidad de Guayaquil. 


\section{Bibliografía}

Abadal, E., Ollé, C., Abad-García, F., \& Melero, R. (2008). El acceso abierto a la ciencia en las universidades españolas. Revista española de documentación científica.

Bates, M. (2007). Attitudes to the rights and rewards for author contributions to repositories for teaching and learning. ALT-J Research in learning technology, 15(1), 67-82.

Caris, M. (2004). Why don't faculty use learning object repositories? Procs. of World conf. on educational multimedia, hypermedia and telecommunications, 2838-2840.

EdReNe. (2009). State of the art II: educational repositories in Europe. Obtenido de http://edrene.org/results/deliverables/EdReNeD3.4TSR_

Ferran, N. (2007). Enriching e-learning metadata through. The electronic library, 25(2), 148-165. Obtenido de http://dx.doi.org/10.1108/02640470710741296

Gadd, E. (2008). A comparison of academics' attitudes towards the rights protection of their research and teaching materials. Active learning in higher education, 9, 103-121.

Harley, D. (2008). Why understanding the use and users of OERs matters. (T. M. Press, Ed.) Opening up education the collective advancement of education through open technology open content and open knowledge, 197-211.

Mortera Gutiérrez, F. J. (2010). Implementación de recursos educativos abiertos (REA) a través del portal TEMOA (Knowledge Hub) del Tecnológico de Monterrey, México. Formación universitaria, 3(5), 920.

OECD. (2007). Giving knowledge for free: the emergence of open. Paris.

Olcos. (2007). Open Educational Practices and Resources: The OLCOS Roadmap 2012. RUSC. Universities and Knowledge Society Journal, 4(1).

OPAL. (2011). Beyond OER: Shifting focus from resources to practices. The OPAL report. Obtenido de http://duepublico.uni-duisburg-essen.de/servlets/Derivate-Servlet/Derivate-25907/OPALReport2011Beyond-OER.pdf

Royster, P. (2011). Review of the survey of institutional digital. Faculty Publications, UNL Libraries. Obtenido de http://digitalcommons.unl.edu/libraryscience/22

SANTOS-HERMOSA, G., FERRÁN-FERRER, N., \& ABADAL, E. (2012). Recursos educativos abiertos: repositorios y uso. El Profesional de la Información, 21(2), 136-145. 